Original Article

\title{
Clinical and Pathological Features of Degenerative Mitral Valve Disease: Billowing Mitral Leaflet Versus Fibroelastic Deficiency
}

\author{
Ichiro Matsumaru, MD, ${ }^{1}$ Kiyoyuki Eishi, MD, PhD,${ }^{1}$ Koji Hashizume, MD,${ }^{1}$ \\ Hiroaki Kawano, MD, PhD, ${ }^{2}$ Akira Tsuneto, $\mathrm{MD}, \mathrm{PhD},{ }^{2}$ \\ and Tomayoshi Hayashi, $\mathrm{MD}, \mathrm{PhD}^{3}$
}

\begin{abstract}
Purpose: Degenerative mitral valve disease is distinguished with billowing mitral leaflet (BML) or fibroelastic deficiency (FED). The purpose of this study is to evaluate the clinical characteristics and the pathohistological differences between BML and FED.

Methods: A total of 73 patients who diagnosed as degenerative mitral valve disease pathologically after mitral valve surgery for severe mitral regurgitation were enrolled. On the basis of echocardiographic features and gross appearances, they were classified as BML ( 9 cases) and FED (64 cases).

Results: In the BML group, multiple segments of the leaflet showed billowing with elongated chordae. Therefore excessive valve tissue needed to be removed by multiple resection and suture. The FED patients had focal myxomatous changes with ruptured chordae, a single resection and suture was frequently employed. In pathological examination, the valve thickness of the BML was nearly twice as thick as the FED, and the mucopolysaccharide accumulation of the Spongiosa in the BML was over $50 \%$, while $30 \%$ in the FED.

Conclusion: BML presents the characteristic valve thickening due to its abnormal production of mucopolysaccharide. Since excessive tissue was voluminous in the BML, high-grade plasty techniques, such as combination of multiple resection and chordal reconstruction were required.
\end{abstract}

Keywords: pathology, mitral valve, mitral regurgitation, mitral valve repair

\section{Introduction}

${ }^{1}$ Department of Cardiovascular Surgery, Nagasaki University Hospital, Nagasaki, Nagasaki, Japan

${ }^{2}$ Department of Cardiovascular Medicine, Nagasaki University Graduate School of Biomedical Science, Nagasaki, Nagasaki, Japan ${ }^{3}$ Department of Pathology, Nagasaki University Hospital, Nagasaki, Nagasaki, Japan

Received: July 3, 2013; Accepted: September 22, 2013

Corresponding author: Ichiro Matsumaru, MD. Department of Cardiovascular Surgery, Sasebo Municipal General Hospital, 9-3

Hirase-machi, Sasebo, Nagasaki 857-8511, Japan

Email: ichiro-m@hospital.sasebo.nagasaki.jp

(C)2014 The Editorial Committee of Annals of Thoracic and Car-

diovascular Surgery. All rights reserved.
Degenerative mitral valve disease is characterized by redundant leaflets prolapsed into the left atrium, chordal elongation or ruptured. It has been suggested that this disease is associated with progressive mitral regurgitation (MR), thrombo-embolism, infective endocarditis, and sudden death. ${ }^{1)}$ Degenerative mitral valve disease is divided into several subtypes according to clinical variability. Carpentier, et al. classified patients with degenerative mitral valve disease into two different forms on the basis of clinical patterns and gross appearance: billowing mitral leaflet (BML), and fibroelastic deficiency (FED), and 
reported that wider degenerative changes were observed in cases of BML. ${ }^{2,3)}$ However, the pathohistological differences have not been clearly defined.

The purpose of our study is to elucidate the pathohistological differences between BML and FED cases using quantitative analysis, as well as to define the clinical characteristics.

\section{Materials and Methods}

\section{Patient background}

A total of 73 patients (40 males and 33 females; average age: $59 \pm 14$ years) who received mitral valve surgery and diagnosed as degenerative mitral valve disease pathologically were enrolled in this study. Patients with Marfan syndrome, rheumatic mitral valve disease, infective endocarditis, and ischemic mitral regurgitation cases were excluded from study participation. All patients provided written informed consent. The use of these tissues was authorized by the hospital's institutional review board (Nagasaki University Hospital, Nagasaki, Japan).

\section{Etiologic classification}

According to the classification of Carpentier, et al., ${ }^{2)}$ we differentiated valvular status between BML and FED on the basis of preoperative echocardiography and the gross appearance as follows: cases exhibiting billowing of the more than one leaflets into the left atrial side ( $1 / 4$ or more of the anterior leaflet [AL] and $1 / 3$ or more of the posterior leaflet [PL]), were categorized into the BML group ( 9 cases, 12\%), and those cases with a limited prolapse ( $1 / 4$ or less of the AL and $1 / 3$ or less of the PL) without excessive tissue were into the FED group (64 cases, $88 \%$ ).

\section{Surgical techniques}

All patients were operated on in the same institution (Nagasaki University Hospital) by an experienced surgeon (K.E). Operations were performed via a full sternotomy under mild hypothermia cardiopulmonary bypass (CPB) with cannulation of the ascending aortic and bicaval cannulation of the superior and inferior vena cavae. After cross-clamping the ascending aorta, a single dose of antegrade cold-crystalloid cardioplegia was injected into the aortic root. Intermittent cold cardioplegia was always used for myocardial protection. The mitral valve was approached through a standard left atriotomy. Meticulous gross examination of the mitral valve apparatus by the surgeon and the presence of leaflets prolapse or chordal rupture were confirmed.
Repair procedures for mitral valve plasty (MVP) were classic techniques previously described by Carpentier, et al. ${ }^{2)}$ Posterior and commissural prolapse were collected by quadrangular, rectangular, or triangular resection and suture with a running or single interrupted suture of 5-0 polypropylene thread (resection/suture technique). If quadrangular resection was performed, horizontal plication sutures were placed to relieve excess tension on the margins of the PL or the commissural lesion. AL prolapse was preferentially gathered by chordal reconstruction, while excessive tissue was collected by chordal replacement $(\mathrm{CR})$ with 5-0 expanded polytetrafluoroethylene (ePTFE) sutures in addition to a small triangular resection and suture. Mitral annuloplasty was done in all cases and the appropriate size was determined by means of standard measurement of the surface of the AL and the commissure-commissure length. Basically, in cases of $\mathrm{AL}$ or BL prolapse, Carpentier-Edwards (C-E) Physio annuloplasty ring (Edwards Lifescience, Irvine, California, USA) was used. In cases of PL prolapse or isolated commissure prolapse, posterior autologous pericardial annuloplasty (PPA) was performed as previously described. ${ }^{4)}$ Before the completion of mitral valve repair, saline testing was performed to confirm the shape and location of the coaptation line.

\section{Pathological studies}

Degenerative mitral valve leaflets and chordae tendineae were submitted to the department of pathology at the Nagasaki University Hospital after surgery. For histopathological examination, the resected segments were immediately fixed in $20 \%$ buffered formalin solution and embedded in paraffin blocks. Following fixation, 2- to 3-mm thick sections were cut from the annular margin to the edge in 2- to 4-mm widths and sliced 4- $\mu \mathrm{m}$ thick to prepare the pathological specimens. Specimens representing the entire transverse area from the annulus to the tip of the leaflets were stained with: Hematoxylin and eosin (HE) and Elastic van Gieson's stain used for general morphology, and Alcian blue (A. blue) for detection of mucopolysaccharide. These specimens underwent microscopic diagnostic evaluation by an experienced pathologist (T.H.)

For further investigations, stained specimens were photographed with an Olympus Digital Camera DP70 (Olympus Corporation; Tokyo, Japan) via an upright microscope (Olympus BX51, Olympus Corporation) and stored as $\times 40$ magnified image data at $2040 \times 1536$ resolution. For displaying measured data and image 
processing, image analysis software Win ROOF (Win ROOF Version 6.1, Mitani Corporation; Fukui, Japan) was employed. The degree of myxomatous degeneration was quantified according to the following methods:

(1) Valve thickness - Valve thickness was calculated from the average of three thickness measurement points (one taken at the junction between the rough zone and clear zone, the second at the mid-position of the clear zone, and the third at the junction between the clear zone and the basal zone.

(2) Percentage of the Spongiosa - We determined the grade of mucopolysaccharide accumulation by estimating the percentage of the Spongiosa for the entire valve thickness in the clear zone. To calculate the area of the Spongiosa, the blue-stained lesion obtained by A. blue staining was employed, as myxomatous degeneration involves retention of acid mucopolysaccharide specifically stained dark blue with A. blue. Briefly, the software captured the blue signal on the samples, and the total area was automatically calculated by WinROOF using the color extraction function.

\section{Echocardiographic assessment and postoperative follow-up}

Images were obtained using two-dimensional and Doppler TTE with a 2.5-MHz transducer (Toshiba; Tokyo, Japan). Color flow was applied in the parasternal and apical views to aid in evaluating mitral regurgitation. The presence and quantity of MR was evaluated by measuring colored areas of jet regurgitation on the image. MR severity was graded as none $(0 / 4$, if regurgitant area was not detected), trivial $\left(0.5 / 4,<2.0 \mathrm{~cm}^{2}\right)$, mild $(1 / 4$, 2-4 $\left.\mathrm{cm}^{2}\right)$, mild to moderate $\left(2 / 4,4-8 \mathrm{~cm}^{2}\right)$, moderate $\left(3 / 4,8-12 \mathrm{~cm}^{2}\right)$, or severe $\left(4 / 4,>12 \mathrm{~cm}^{2}\right)$, and a regurgitation jet area $8 \mathrm{~cm}^{2}$ or larger was defined as a significant recurrent MR.

In all cases, intraoperative trans-esophageal echocardiography (TEE) was performed after introduction of general anesthesia. At the time of CPB weaning, TEE was performed to assess the degree of residual MR. If the maximum regurgitant jet area exceeded $2.0 \mathrm{~cm}^{2}$, intraoperative redo was conducted. The condition for the completion of repair was the presence of a regurgitant area $<2.0 \mathrm{~cm}^{2}$. Postoperative TTE was performed at 2 weeks after surgery and periodically, every 6 to 12 months after discharge. All echocardiography were assessed in the core cardiology unit by an experimental cardiologist (A.T.). Patients were followed by echocardiography for a mean of $4.8 \pm 3.3$ years (range $0.17-13.0$ years). In total, the clinical follow-up represents. The average followup period was $5.8 \pm 3.2$ years (range $0.83-13.0$ years).

\section{Statistical analysis}

Continuous data were expressed either as a percentage of the total or as a mean \pm 1 standard deviation. Student's $t$ test (two-sided) and the $\chi^{2}$ test were employed for statistical analysis and difference with $\mathrm{p}<0.05$ was considered significant.

Statistical analyses were performed using Stat Mate (version 4.01; ATMS Co., Ltd., Tokyo, Japan).

\section{Results}

\section{Preoperative data}

Preoperative patient demographic data and mitral valve characteristics in both groups are listed in Table 1. There were 9 cases in the BML group ( 4 men and 5 women; mean age: $49 \pm 16$ years) and 64 cases in the FED group (36 men and 28 women; mean age: $60 \pm 13$ years); patients were significantly younger in the BML group than in the FED group. There was no statistically significant difference between these groups about cardiac function, and the NYHA functional class. In the BML group, all cases showed prolapse of BL, while in the FED group, there were 12 cases (19\%) with AL, 46 (72\%) with PL, and $6(9 \%)$ with prolapse of BL. As to the status of chordae tendineae, the mean numbers of leaflet segment with torn chordae were more frequently in the FED group (1.03 \pm 0.44$)$ than in the BML group $(0.33 \pm 0.50)$. On the other hand, the mean numbers of leaflet segment with chordal elongation or thickness were more frequently in the BML group $(3.0 \pm 2.29)$ than in the FED group $(0.16 \pm 0.41)$.

\section{Intraoperative data}

Surgical data are listed in Table 2. There was 1 MVR case $(11 \%)$ and 8 MVP cases (89\%) in the BML group, compared with 2 MVR (3\%) and 62 MVP cases (97\%) in the FED group. The indications showing preference to MVR above MVP were as follows: 1 . Severe anterior mitral leaflet involvement, 2. Severe calcification of the mitral annulus, and 3. Concomitant aortic valve replacement. With respect to the plasty techniques, resection/ suture or plication sites numbers was $1.75 \pm 0.89$ in the BML group and $1.10 \pm 0.59$ in the FED group $(\mathrm{p}<0.01)$, and the CR sites numbers was $0.88 \pm 1.36$ in the BML group and $0.21 \pm 0.45$ in the FED group $(p<0.01)$. Compared to the FED group, the BML group required a more 
Table 1 Demographic data and mitral valve characteristics

\begin{tabular}{lccc}
\hline & BML $(\mathrm{n}=9)$ & FED $(\mathrm{n}=64)$ & $\mathrm{p}$-value \\
\hline Age (years) & $49 \pm 16$ & $60 \pm 13$ & $<0.05$ \\
Male (no.) & $4(50 \%)$ & $36(56 \%)$ & $\mathrm{NS}$ \\
NYHA Class & & & $\mathrm{NS}$ \\
$\quad$ I & $3(33 \%)$ & $10(16 \%)$ & \\
II & $5(56 \%)$ & $33(52 \%)$ & \\
III & $1(11 \%)$ & $19(30 \%)$ & \\
IV & 0 & $1(2 \%)$ & \\
Preoperative AF & $2(22 \%)$ & $17(27 \%)$ & $\mathrm{NS}$ \\
LVEF (\%) & $71 \pm 7.6$ & $72 \pm 9.7$ & $\mathrm{NS}$ \\
Leaflet involvement & & & $<0.001$ \\
$\quad$ AL & 0 & $12(19 \%)$ & \\
PL & 0 & $46(72 \%)$ & \\
$\quad$ BL & $9(100 \%)$ & $6(9 \%)$ & \\
Chordae condition & & & \\
Torn chordae (mean) & $0.33 \pm 0.50$ & $1.03 \pm 0.44$ & $<0.001$ \\
Elongation or Thickening (mean) & $3.0 \pm 2.29$ & $0.16 \pm 0.41$ & $<0.001$ \\
\hline
\end{tabular}

BML: billowing mitral leaflet; FED: fibroelastic deficiency; NYHA: New York Heart Association; AF: atrial fibrillation; LVEF: left ventricular ejection fraction; AL: anterior leaflet; PL: posterior leaflet; BL: bileaflet

Table 2 Surgical data

\begin{tabular}{lccc}
\hline & BML $(\mathrm{n}=9)$ & FED $(\mathrm{n}=64)$ & p-value \\
\hline MVP & $8(89 \%)$ & $62(97 \%)$ & NS \\
Repair techniques & BML $(\mathrm{n}=8)$ & FED $(\mathrm{n}=62)$ & \\
$\quad$ Segmental resection & $1.75 \pm 0.89$ & $1.10 \pm 0.59$ & $<0.01$ \\
$\quad$ or plication sites (n) & & & \\
$\quad$ CR sites (n) & $0.88 \pm 1.36$ & $0.21 \pm 0.45$ & $<0.01$ \\
Annuloplasty & & & $<0.05$ \\
$\quad$ C-E Physio-ring & $6(75 \%)$ & $18(29 \%)$ & \\
PPA & $2(25 \%)$ & $44(71 \%)$ & \\
Ring size (mm) & $30.5 \pm 2.3$ & $28.7 \pm 1.8$ & $<0.05$ \\
Op time (min) & $219 \pm 61$ & $213 \pm 41$ & $\mathrm{NS}$ \\
CPB time (min) & $123 \pm 57$ & $103 \pm 29$ & $\mathrm{NS}$ \\
AoX time (min) & $87 \pm 51$ & $62 \pm 21$ & $<0.05$ \\
\hline
\end{tabular}

BML: billowing mitral leaflet; FED: fibroelastic deficiency; MVP: mitral valve plasty; CR: chordal replacement; C-E Physio-ring: Carpentier-Edwards physio annuloplasty ring; PPA: posterior autologous pericardial annuloplasty; Op: operative; CPB: cardiopulmonary bypass; AoX: aortic cross-clamp

complex plasty technique. In the BML group, 6 patients received a C-E Physio annuloplasty ring and 2 patients received PPA. In the FED group, 44 patients received PPA and 18 patients received a C-E Physio annuloplasty ring. It appears that the rings size was significantly larger in the BML group than in the FED group. The aortic cross-clamp times were significantly longer in the BML group, but there was no significant difference in operation and $\mathrm{CPB}$ time.

\section{Clinical outcome and morbidity}

There was no in-hospital mortality in the each group. Of the 73 patients, 2 patients $(2.7 \%)$ in the FED group were lost to follow-up. There was no long-term mortality in the BML group, but 7 cases were reported for the FED group (cardiac death: 1; non-cardiac death: 6). Six patients (1 BML. 5 FED) had recurrent moderate to severe MR after MVP. Of the 6 patients, 2 (1 BML, 1 FED) showed early recurrence moderate or severe MR within 6 months after surgery and received MVR. Patient in the BML 
Table 3 Pathological findings and Correlation with surgical outcome

\begin{tabular}{lccc}
\hline & BML $(\mathrm{n}=9)$ & FED $(\mathrm{n}=64)$ & $\mathrm{p}$-value \\
\hline Valve Thickness $(\mu \mathrm{m})$ & $2458 \pm 1356$ & $1345 \pm 682$ & $<0.001$ \\
The spongiosa $(\%)$ & $58 \pm 17 \%$ & $31 \pm 18 \%$ & $<0.001$ \\
\hline
\end{tabular}

BML: billowing mitral leaflet; FED: fibroelastic deficiency

group had an extreme case of Barlow's disease, and had received multiple resection/suture technique and chordal shorting and CR with ePTFE suture and PPA. According to the reoperation findings, the cause of MR was the ineffectiveness of the artificial chords and annular dilatation in spite of PPA. Patient in the FED group had a dehiscence of the resection/sutures segment. The other 4 patients in the FED group, showed recurrent MR at $1.7 \mathrm{y}, 3.4 \mathrm{y}, 8.0 \mathrm{y}, 9.5 \mathrm{y}$ after operation, but they are under medical control because they haven't shown the obvious sign of heart failure or rejected the re-do surgery.

\section{Pathohistological finding and correlation with surgical outcome}

Table 3 shows the comparison of histological characteristics in the two groups. Valve thickness was $2458 \pm$ $1356.1 \mu \mathrm{m}$ in the BML group and $1332 \pm 685.8 \mu \mathrm{m}$ in the FED group $(\mathrm{p}<0.001)$, and the valve was significantly thicker in the BML group. The percentage of the Spongiosa was $58 \pm 17.1 \%$ in the BML group and $31 \pm 17.9 \%$ in the FED group $(\mathrm{p}=0.002)$, and the percentage of the Spongiosa were significantly higher in the BML group. In the BML group, diffuse mucopolysaccharide infiltration was widely spread in the spongiosa layer (Fig. 1A). Meanwhile, in the FED group, resected tissue is usually thinner than in BML patients and its four-layer architecture of the leaflet tissue is almost preserved (Fig. 1B).

\section{Discussion}

\section{Normal mitral valve structure and myxomatous degeneration}

Normal mitral valves leaflets have four well-defined tissue layers, from the atrial to the ventricular aspect, these layers were the Auricularis, the Spongiosa, the Fibrosa, and the Ventricularis. Each layer, containing characteristic cells and extracellular matrix, plays a different role.5) Although histologic alteration in myxomatous valves has been well described in the past, ${ }^{6}$ only recently have mechanical properties of myxomatous mitral valves been extensively studied ${ }^{7)}$ and it is shown that myxomatous leaflets are more extensive and less stiff than normal valves. The main pathological hallmarks in degenerative mitral
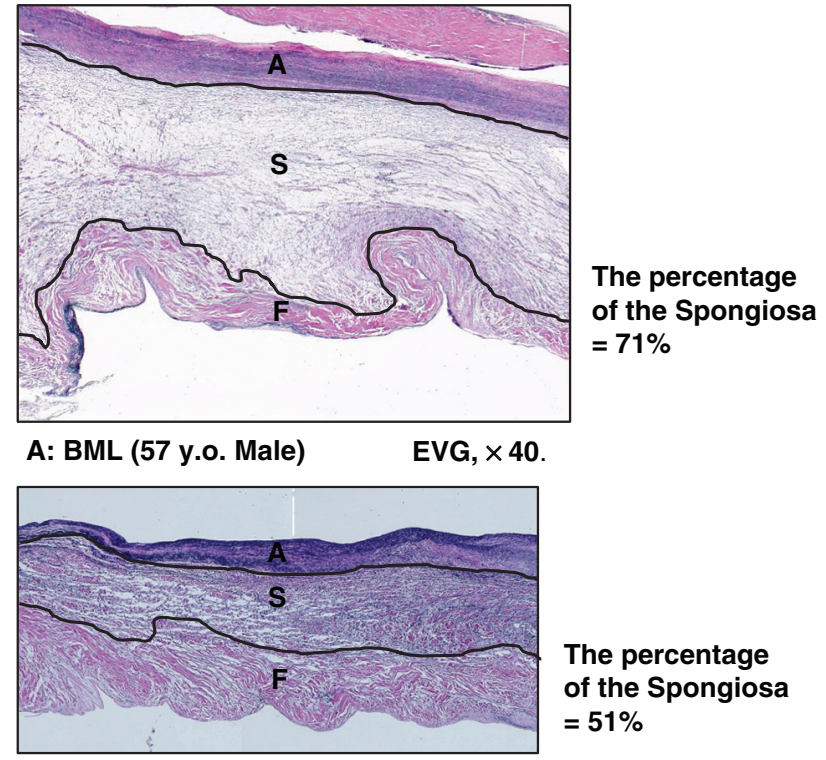

The percentage of the Spongiosa $=51 \%$

\section{B: FED (47 y.o. Male) EVG, $\times 40$.}

Fig. 1 (A) Histologic sections of BML. Mucopolysaccharide were widely infiltrating into the Spongiosa, which thickened the valve in the BML group, giving the appearance of cystic spaces (Elastic van Gieson's stain, original magnification $\times 40$.) $($ B $)$ Histologic sections of FED. In the FED group, resected tissue is usually thinner than in BML and its four-layer architecture of the leaflet tissue is almost preserved. (Elastic van Gieson's stain, original magnification $\times 40$.) A: Auricularis; S: Spongiosa; F: Fibrosa.

valve disease are abnormal accumulations of mucopolysaccharide in the spongiosa, and infiltration to the Fibrosa as a structural core for valve tissue. The layered architecture is destroyed and valve thickening and structural fragility are induced, because individual collagen bundles were fragmented, coiled, disrupted, and decreased consistency is derived from the complicated structure. ${ }^{3,6,8-10)}$ In addition, it has been reported that similar changes are observed in chordal tendineae. ${ }^{11-13)}$

In some cases, there is an identified genetic and congenital defect in the connective tissue, such as in Marfan syndrome. ${ }^{14-17)}$ Recent studies have demonstrated that mucopolysaccharide was abnormally accumulated and immune activity against extracellular matrix proteins 
(such as fibrin and elastin ${ }^{18)}$ and collagen I and III ${ }^{19)}$ ). Rabkins, et al. hypothesized that activated metalloproteinase (MMP) in valvular interstitial cells mediates extracellular matrix degeneration in cases with myxomatous degeneration. ${ }^{20)}$ Mahimkara, et al. demonstrated that increased activity of MMP-2 in transgenic mice reproduces many of the features of human degenerative mitral valve disease. ${ }^{21}$ )

\section{Clinical characteristics of degenerative mitral valve disease}

In this study, BML patients are more likely to be middleaged, and they have a long-term evolution of mitral valve insufficiency (10-20 years), because they had been found to have a cardiac murmur on physical examination when they were young, but often had no symptoms. In the BML group, multiple segments of the leaflet showed billowing into the atrial side with redundant tissue and its chordae are typically elongated, thickened, and fused. Therefore excessive valve tissue needed to be removed by multiple resection/suture techniques. Moreover, since an AL lesion was included, a combination procedure, with $\mathrm{CR}$ was necessary after reduction of excess tissue. We also confirmed that patients with BML had a significantly more dilated annulus than those with FED, and the use of large annuloplasty with a total ring to prevent the complication of systolic anterior motion was needed.

On the other hand, there were some elderly patients in the FED group who did not have a long history of a murmur. Their disease duration tended to be shorter than the in the BML group. This might be a reflection of the fact that FED patients presented frequently with ruptured chordae. Compared with the BML group, the FED patients has focal myxomatous changes with a restriction of thickening to the limited prolapsing segment, sometimes with visible ruptured chordae; the remaining valve tissue had a more pellucid aspect, and the annulus was not dilated or was only slightly dilated. There was less excessive tissue, and a single resection/suture technique was most frequently employed in the FED group. Since the annulus was not enlarged or only slightly enlarged, PPA alone successfully prevented regurgitation.

Most reports in the mitral valve repair literature define patient subsets on the basis of leaflet dysfunction and repair techniques, whereas the etiology of degenerative disease has been rarely described. Adams, et al. addressed the importance of the difference in etiology of lesions with myxomatous degeneration. ${ }^{22)}$ Flameng, et al. assessed serial echocardiographic follow-up of valve function, including the regurgitation recurrence-free rate, after plasty for Barlow's disease and FED, and reported that the etiology of myxomatous degeneration influenced the complexity of plasty techniques and postoperative valve regurgitation. ${ }^{23)}$

Especially if try to repair for BML, so called the extreme Barlow's disease showing billowing and prolapse of BL, is often difficult because it has excessive leaflet tissue characterized by myxomatous degeneration. We performed repair in an extreme Barlow's disease patient with CS for all segments, $8 \mathrm{CR}$ with ePTFE sutures for AL and $2 \mathrm{CR}$ for PL, resection/suture of the clear zone of PL, and a 34-mm posterior annuloplasty with autologous pericardium. The residual MR jet area at the end of surgery was $<1.5 \mathrm{~cm}^{2}$. However, 6 months after surgery, there was recurrent moderate MR due to the ineffectiveness of the artificial chords in AL. We hypothesized that the absence of a reference point to determine the appropriate length of the artificial chords, the residual redundant tissue in $\mathrm{AL}$, and partial annuloplasty might be the cause of recurrent MR.

\section{Pathohistological change of degenerative mitral valve disease}

McDonald, et al. measured the thickness of normal mitral valve leaflets and showed the average thickness of clear zone was $0.7-0.9 \mathrm{~mm} .{ }^{5)}$ Olsen, et al. examined the range of the extent of the spongiosa and reported that the percentage of the Spongiosa in relation to the valve thickness was variable but almost $10 \%-20 \%$ in the normal valve and over $60 \%$ in floppy valve syndrome. ${ }^{24)}$

In this study, we demonstrated that there was a distinct difference between these two entities by measurement of valve thickness and quantification of the contained mucopolysaccharide. The valve thickness of BML was thicker than FED $(2458 \pm 1356.1 \mu \mathrm{m}$ vs. $1332 \pm 685.8 \mu \mathrm{m})$ and the proportion of the Spongiosa in the BML group was over 50\%, while 30\% in the FED group. Compared with the FED group, mucopolysaccharide were widely infiltrating not only into the Spongiosa but also into the space between collagen fibers in the Fibrosa, which thickened the valve in the BML group, giving the appearance of cystic spaces. Meanwhile, in the FED group, resected tissue is usually thinner than in BML patients because it has less myxomatous infiltration and its fourlayer architecture of the leaflet tissue is almost preserved. The high incidence of chordal rupture suggests the first mechanism and secondary pathological change in prolapsing segments may result in myxoid deposition with 
resulting thickening and expansion, but the process is usually limited in the prolapsing segment.

\section{Study Limitations}

It should be noted that only resected valvular segments were investigated in our study.

In BML, affected sites were resected widely, but only the leaflet might have been resected in FED. Therefore, it was possible that the whole pathohistological change was not evaluated. Moreover, since some cases underwent plasty without resection of valve tissue, it was possible that cases with mild degeneration were not included.

\section{Conclusion}

Compared with the FED group, BML presents the characteristic valve thickening due to its abnormal production of mucopolysaccharide. In the BML group, excessive valve tissue needed to be removed by multiple resection and suture techniques with combination procedures and the use of large annuloplasty. On the other hand, there was less excessive tissue in the FED group, and a single resection and suture technique was most frequently employed. Since the annulus was not enlarged or only slightly enlarged, posterior pericardial annuloplasty alone successfully prevented regurgitation.

\section{Acknowledgement}

We thank the technical staff of the Department of Pathology, Nagasaki University Hospital for their assistance in sectioning and staining the valves.

\section{Disclosure Statement}

We have no financial support or conflicts of interest to declare in relation to this study.

\section{References}

1) Barlow JB, Pocock WA. Mitral valve billowing and prolapse: perspective at 25 years. Herz 1988; 13: 227-34.

2) Carpentier A, Chauvaud S, Fabiani JN, et al. Reconstructive surgery of mitral valve incompetence: tenyear appraisal. J Thorac Cardiovasc Surg 1980; 79: $338-48$.

3) Fornes P, Heudes D, Fuzellier JF, et al. Correlation between clinical and histologic patterns of degener- ative mitral valve insufficiency: a histomorphometric study of 130 excised segments. Cardiovasc Pathol 1999; 8: 81-92.

4) Matsukuma S, Eishi K, Yamachika S, et al. Risk factors of posterior pericardial annuloplasty for isolated posterior leaflet prolapse. Ann Thorac Surg 2005; 80: 820-4.

5) McDonald PC, Wilson JE, McNeill S, et al. The challenge of defining normality for human mitral and aortic valves: geometrical and compositional analysis. Cardiovasc Pathol 2002; 11: 193-209.

6) Tamura K, Fukuda Y, Ishizaki M, et al. Abnormalities in elastic fibers and other connective-tissue components of floppy mitral valve. Am Heart J 1995; 129: 1149-58.

7) Barber JE, Kasper FK, Ratliff NB, et al. Mechanical properties of myxomatous mitral valves. J Thorac Cardiovasc Surg 2001; 122: 955-62.

8) Gupta V, Barzilla JE, Mendez JS, et al. Abundance and location of proteoglycans and hyaluronan within normal and myxomatous mitral valves. Cardiovasc Pathol 2009; 18: 191-7.

9) Grande-Allen KJ, Griffin BP, Ratliff NB, et al. Glycosaminoglycan profiles of myxomatous mitral leaflets and chordae parallel the severity of mechanical alterations. J Am Coll Cardiol 2003; 42: 271-7.

10) Lis Y, Burleigh MC, Parker DJ, et al. Biochemical characterization of individual normal, floppy and rheumatic human mitral valves. Biochem J 1987; 244: 597-603.

11) Akhtar S, Meek KM, James V. Ultrastructure abnormalities in proteoglycans, collagen fibrils, and elastic fibers in normal and myxomatous mitral valve chordae tendineae. Cardiovasc Pathol 1999; 8: 191-201.

12) Baker PB, Bansal G, Boudoulas H, et al. Floppy mitral valve chordae tendineae: histopathologic alterations. Hum Pathol 1988; 19: 507-12.

13) Scott-Jupp W, Barnett NL, Gallagher PJ, et al. Ultrastructural changes in spontaneous rupture of mitral chordae tendineae. J Pathol 1981; 133: 185-201.

14) Nesta F, Leyne M, Yosefy C, et al. New locus for autosomal dominant mitral valve prolapse on chromosome 13: clinical insights from genetic studies. Circulation 2005; 112: 2022-30.

15) Trochu JN, Kyndt F, Schott JJ, et al. Clinical characteristics of a familial inherited myxomatous valvular dystrophy mapped to Xq28. J Am Coll Cardiol 2000; 35: 1890-7.

16) Segura AM, Luna RE, Horiba K, et al. Immunohistochemistry of matrix metalloproteinases and their inhibitors in thoracic aortic aneurysms and aortic valves of patients with Marfan's syndrome. Circulation 1998; 98: II331-7; discussion II337-8.

17) Read RC, Thal AP, Wendt VE. Symptomatic valvular myxomatous transformation (the floppy valve syndrome). A possible forme fruste of the Marfan syndrome. Circulation 1965; 32: 897-910.

18) Nasuti JF, Zhang PJ, Feldman MD, et al. Fibrillin and 
other matrix proteins in mitral valve prolapse syndrome. Ann Thorac Surg 2004; 77: 532-6.

19) Cole WG, Chan D, Hickey AJ, et al. Collagen composition of normal and myxomatous human mitral heart valves. Biochem J 1984; 219: 451-60.

20) Rabkin E, Aikawa M, Stone JR, et al. Activated interstitial myofibroblasts express catabolic enzymes and mediate matrix remodeling in myxomatous heart valves. Circulation 2001; 104: 2525-32.

21) Mahimkar R, Nguyen A, Mann M, et al. Cardiac transgenic matrix metalloproteinase-2 expression induces myxomatous valve degeneration: a potential model of mitral valve prolapse disease. Cardiovasc Pathol 2009; 18: 253-61.

22) Adams DH, Anyanwu AC. Seeking a higher standard for degenerative mitral valve repair: begin with etiology. J Thorac Cardiovasc Surg 2008; 136: 551-6.

23) Flameng W, Meuris B, Herijgers P, et al. Durability of mitral valve repair in Barlow disease versus fibroelastic deficiency. J Thorac Cardiovasc Surg 2008; 135: 274-82.

24) Olsen EG, Al-Rufaie HK. The floppy mitral valve. Study on pathogenesis. Br Heart J 1980; 44: 674-83. 